BMC

Dermatology

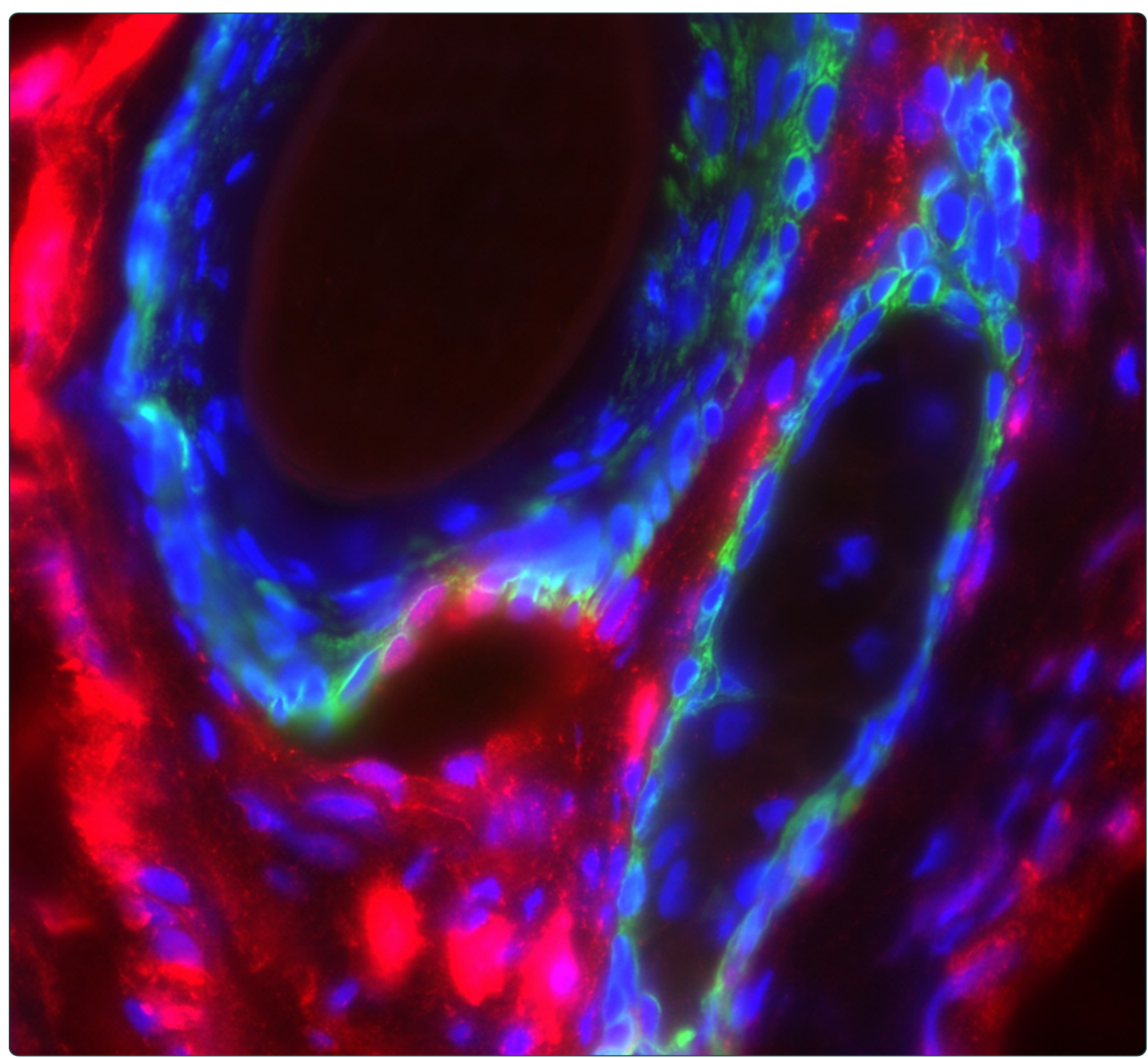

TGF $\beta$ signaling regulates lipogenesis in human sebaceous glands cells

McNairn et al.

C Biomed Central 


\title{
TGF $\beta$ signaling regulates lipogenesis in human sebaceous glands cells
}

\author{
Adrian J McNairn ${ }^{1,2 \dagger}$, Yanne Doucet ${ }^{1,3 \dagger}$, Julien Demaude ${ }^{4}$, Marion Brusadelli ${ }^{1}$, Christopher B Gordon ${ }^{5}$, \\ Armando Uribe-Rivera ${ }^{5}$, Paul F Lambert ${ }^{6}$, Charbel Bouez ${ }^{4}$, Lionel Breton ${ }^{4}$ and Géraldine Guasch ${ }^{1 *}$
}

\begin{abstract}
Background: Sebaceous glands are components of the skin essential for its normal lubrication by the production of sebum. This contributes to skin health and more importantly is crucial for the skin barrier function. A mechanistic understanding of sebaceous gland cells growth and differentiation has lagged behind that for keratinocytes, partly because of a lack of an in vitro model that can be used for experimental manipulation.

Methods: We have developed an in vitro culture model to isolate and grow primary human sebocytes without transformation that display functional characteristics of sebocytes. We used this novel method to probe the effect of Transforming Growth Factor $\beta$ (TGF $\beta$ ) signaling on sebocyte differentiation, by examining the expression of genes involved in lipogenesis upon treatment with TGF $\beta 1$. We also repressed TGF $\beta$ signaling through knockdown of the TGF $\beta$ Receptor // to address if the effect of TGF $\beta$ activation is mediated via canonical Smad signal transduction.

Results: We find that activation of the TGF $\beta$ signaling pathway is necessary and sufficient for maintaining sebocytes in an undifferentiated state. The presence of TGF $\beta$ ligand triggered decreased expression in genes required for the production of characteristics sebaceous lipids and for sebocyte differentiation such as FADS2 and PPARY, thereby decreasing lipid accumulation through the TGF $\beta$ RII-Smad2 dependent pathway.

Conclusion: TGF $\beta$ signaling plays an essential role in sebaceous gland regulation by maintaining sebocytes in an undifferentiated state. This data was generated using a novel method for human sebocyte culture, which is likely to prove generally useful in investigations of sebaceous gland growth and differentiation. These findings open a new paradigm in human skin biology with important implications for skin therapies.
\end{abstract}

Keywords: Human Sebaceous gland cells, Sebocytes, TGF $\beta$ signaling, Cell differentiation, Proliferation, Lipogenesis, Skin appendages

\section{Background}

In humans, sebaceous glands associated with hair follicles are distributed throughout all the skin and found in greatest abundance on the face and scalp and are absent from the palms and soles [1]. Sebaceous glands can also form independently from the hair follicle and form specialized glands such as Meibomian glands of the eyelid, ectopic sebaceous gland of the glans penis [2] and Fordyce's spots of the oral epithelium. Sebaceous glands are microscopic glands which secrete an oily substance

\footnotetext{
* Correspondence: geraldine.guasch@cchmc.org

†Equal contributors

'Division of Developmental Biology, Cincinnati Children's Hospital Medical Center, 3333 Burnet Avenue, Cincinnati, OH 45229, USA

Full list of author information is available at the end of the article
}

(sebum) in the hair follicles to lubricate the skin and hair of animals [3]. Their function within the epidermis is to prevent the skin from dehydration and protect the body against infections and physical, chemical and thermal assaults of the environment. The main components of human sebum are triglycerides and fatty acids (57.5\%), wax esters (26\%), and squalene (12\%) [4]. The production of sebum is regulated throughout life, and decreases dramatically with age [5]. This is associated with increased dryness and fragility of the skin. Moreover, several human diseases, such as acne vulgaris, atopic dermatitis, seborrheic dermatitis and primary cicatricial alopecia are thought to be associated with deregulation of the sebaceous glands $[4,6,7]$.

\section{Biomed Central}


There is a crucial interdependency of sebaceous glands with hair follicles and epidermis as sebocyte dysfunction results in degeneration of hair follicle structures and a defective skin barrier $[7,8]$. This is illustrated in the asebia mutant mouse, which lacks the SCD1 enzyme that desaturates fatty acids. This mutant displays rudimentary sebaceous glands and alteration in the profile of skin surface lipids leading to chronic inflammatory reactions, alopecia and dermal scarring [8].

Successful growth of primary human cells often constitutes a breakthrough in a specific area of human biology with important clinical implications. Tissue stem cells such as those of the blood and the epidermis have already been successfully used in clinics for decades $[9,10]$. In particular, epidermal cells (keratinocytes) can be cultured in vitro and can be efficiently manipulated to form a three dimensional epidermis $[11,12]$. Despite these advancements, the successful methods for culturing human primary sebocytes without the use of mouse feeder layers are not established. Selective cultivation of human sebocytes has been attempted in the past using mitomycin-treated 3T3 feeder layers by covering the microdissected sebaceous gland explant with glass slides but primary sebocytes survived only two passages after which they underwent differentiation [13]. Human sebaceous gland cell lines have been established in the past from adult human facial skin and periauricular area [14-17], but their immortalization with Simian virus-40 large T antigen or HPV16/E6E7 genes, which bypass the p53 and retinoblastoma protein mediated restriction point, results in cellular transformation that has limited their use for analyzing their cell cycle and differentiation regulation. Here, we culture human primary sebocytes using a novel method, which can in the future, be incorporated into skin reconstructs and provide a basis for understanding the molecular pathways which regulate human sebaceous gland biology.

A potential candidate for human sebocyte regulation suggested by several lines of evidence is Transforming Growth Factor $\beta$ (TGF $\beta$ ) $[18,19]$ but the lack of primary human cultures has impaired an in-depth investigation of the molecular mechanism whereby TGF $\beta$ signaling controls sebaceous gland differentiation. The TGF $\beta$ pathway is ubiquitous and involved in the control of growth and differentiation of multiple cell and tissue types. The two major receptors of the TGF $\beta$ signaling pathway, TGF $\beta$ Receptor I (TGF $\beta$ RI) and TGF $\beta$ Receptor II (TGF $\beta$ RII), are expressed in mouse sebaceous glands [20,21]. In human and mouse epithelial cell lines, TGF $\beta$ acts as a potent inhibitor of proliferation mediated at least in part via down-regulation of c-Myc expression [22,23]. Intriguingly, c-Myc overexpression in a mouse model induces an increase in sebaceous gland size due to activation of sebocyte differentiation at the expense of hair differentiation [16,24].
Moreover, disruption of epidermal Smad4, the common mediator of TGF $\beta$ signaling, leads to hyperplasia of interfollicular epidermis, hair follicle, and sebaceous glands through c-Myc upregulation [25].

To determine the effect of TGF $\beta$ signaling on sebocyte differentiation, we investigated the effect of TGF $\beta$ ligands on the primary human sebocytes we established using a novel culture system and skin samples from pediatric donors.

\section{Results}

\section{Primary sebocytes established from pediatric donors express markers of sebaceous gland differentiation}

To determine the pathways that regulate primary human sebocytes growth and differentiation, we developed a novel culture method by mimicking the microenvironment of the sebaceous glands in vitro. Skin explants from donors ranging from 9 months to 12 years of age were microdissected (Figure 1a-b) and the sebaceous glands were placed between fibronectin-coated glass coverslips to reproduce an in vivo environment (Figure 1c-d). Using this technique, primary sebocyte cultures were derived from eight donors representing four skin tissue types: five scalp, one breast, one chest, and one face sample. While this technique enabled us to continually passage sebocytes beyond 15 passages, all experiments were performed on passage 2 and later passages ( 3 to 5 ) without the use of extracellular matrix or supporting irradiated fibroblasts.

To verify that the cell cultures were indeed sebocytes, we examined the expression of known sebocyte markers. Immunofluorescence staining and immunoblot demonstrated that those cells homogeneously express peroxisome proliferator-activated receptor gamma (PPAR $\gamma$ ) an adipogenic transcription factor expressed in differentiating sebocytes [26], in vitro (Figure 1d and Figure 2a, Scalp-derived Sebaceous Gland cells SSG3) and in vivo (Additional file 1: Figure S1a-b) but not in human keratinocytes (NIKS) [27]. Real-time PCR confirmed that primary SSG3 expressed a similar level of PPAR $\gamma$ as the immortalized sebocyte line SEB-1 [15] (Figure 2b). However, SEB-1 expresses Keratin 8, a protein associated with skin appendages tumors [28], whereas SSG3 cells do not express Keratin 8 (Figure 2a), akin to sebaceous gland in vivo [29]. Additionally, SSG3 cells express other markers of sebocytes such as Blimp1 and epithelial membrane antigen EMA/Muc1 (Additional file 1: Figure S1c, d and e). In agreement with recent reports [16,30], Blimp1 is expressed in the inner root sheath of the hair follicle and in terminally differentiated cells of the sebaceous glands in human scalp sections from which SSG3 cells were derived (Additional file 1: Figure S1c). All the results shown in scalp-derived sebocytes have been confirmed to be similar in the breast, chest and face derived-sebocytes (Additional file 1: Figure S1g). The only 

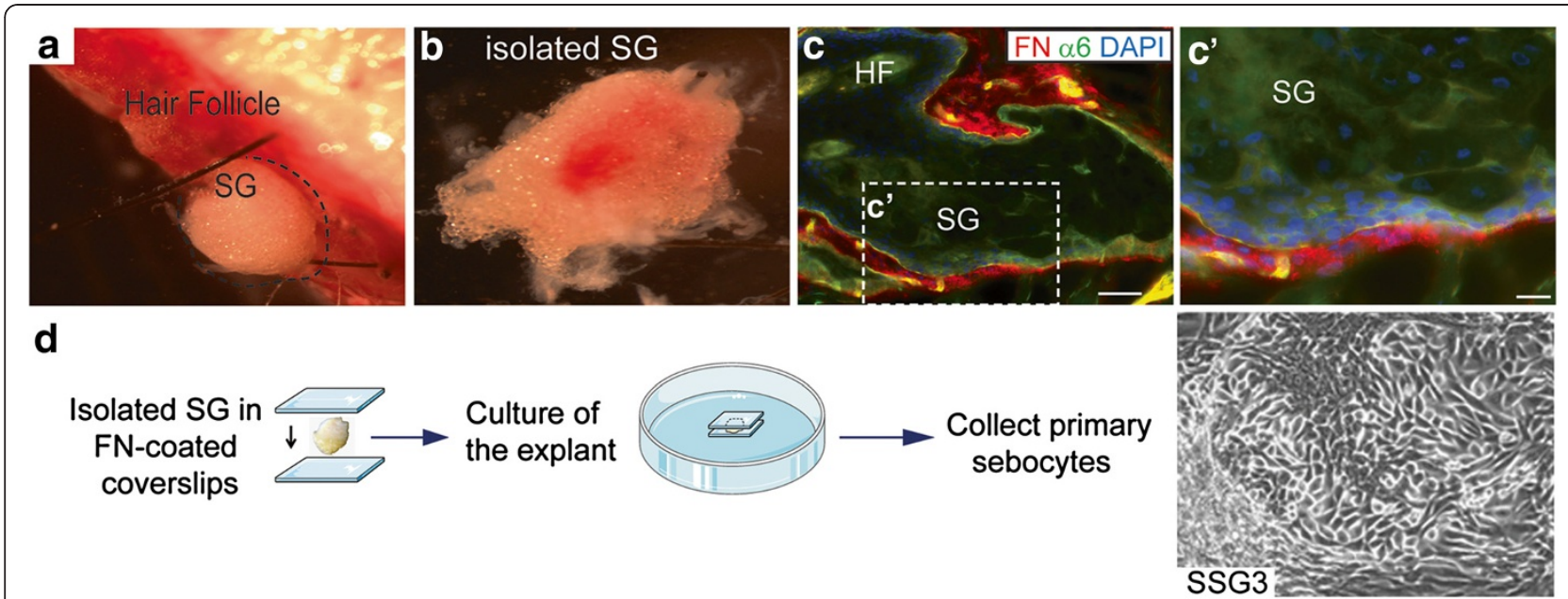

Figure 1 Fibronectin mimics the microenvironment and allows sebocytes to grow in vitro. (a) Scalp sample ( 9 months old) before microdissection. (b) Isolated sebaceous gland. (c) Immunofluorescence staining on OCT sections of human scalp tissue showed that fibronectin (in red) is expressed in the extracellular matrix surrounding the sebaceous gland. a6-integrin (in green) marked the basal layer of the gland. Boxed area is magnified and shown to ( $\left.\mathbf{c}^{\prime}\right)$. Scale bars, $20 \mu \mathrm{m}\left(\mathbf{c}, \mathbf{c}^{\prime}\right)$. (d) Schematic of new method to isolate and cultivate sebocytes. Scalp explants were placed between coverslips coated with fibronectin. Sebaceous gland cells SSG3 growing out of the explant (100x magnification). Abbreviations: SG, Sebaceous Gland, HF, Hair Follicle, FN, Fibronectin.

exception is the expression of Keratin 7, a marker of the undifferentiated sebocytes, detected at higher expression in protein lysates of the face-derived sebocytes compared to the scalp, the breast and the chest (Additional file 1: Figure S1f-g). The difference in Keratin 7 expression may depend on the location from which the cells derived (Additional file 1: Figure S1d). To conclude, we have established primary human sebocytes that express typical sebocyte markers and represent a good model for studying sebocyte function.

\section{Primary sebocytes can differentiate in vitro}

To confirm that the primary human sebocytes are functional in vitro, we analyzed their ability to differentiate and produce human-specific lipids. The lipophilic dye Nile red can be used to stain terminally differentiating sebocytes [31] (Additional file 2: Figure S2a). Linoleic acid is an essential polyunsaturated fatty acid used for biosynthesis of arachidonic acid and other polyunsaturated fatty acids that can trigger the differentiation of sebocytes in vitro [32]. We therefore analyzed the cellular lipid distribution by Nile red after two days of linoleic acid treatment at physiological levels and show that SSG3 produce lipids in response to linoleic acid (Additional file 2: Figure S2b). Moreover, we detected cytosolic lipid droplets by electron microscopy in untreated cells (Additional file 2: Figure S2c) as well as an increase of lipid droplets with higher electron density after linoleic acid treatment (Additional file 2: Figure S2c"). Humans possess a unique $\Delta 6$ desaturase/FADS2 gene [33] involved in linoleic acid metabolism and sebum production. FADS2 is detectable mainly in differentiated sebocytes that have reached lipid synthesis capacity, providing a functional marker of activity and differentiation in sebocytes. We have found that FADS2 is highly expressed in SSG3 cells compared to SEB-1 (Figure 2c). These results demonstrate that the SSG3 cells exhibit gene expression patterns characteristics of cells involved in sebocyte differentiation. Moreover, we found that the differentiation induced by linoleic acid treatment in SSG3 cells is followed by an increase in PPAR $y$ at $48 \mathrm{~h}$ (Figure 2d) and an increase of FADS2 after $24 \mathrm{~h}$ and $48 \mathrm{~h}$ of treatment when cells have reached a high level of cytoplasmic lipid production (Figure 2e).

To further confirm the presence of human specific lipids, gas chromatography of SSG3 cells was performed. We found differences in the composition of fatty acids, in particular, sapienic acid, predominantly found in sebum in vivo [33], and palmitoleic acid. They are synthesized by two desaturases, $\Delta 6 /$ FADS2 and $\Delta 9$ respectively [34] (Figure 2f). The desaturation in $\Delta 6$ position instead of $\Delta 9$ is specific to human sebum [34]. Sapienic acid is detected only in SSG3 cells $(2.150 \%)$ compared to NIKS $(0.795 \%)$. In contrast, palmitoleic acid is predominantly found in NIKS (6.959\%) compared to SSG3 cells (1.202\%) (Figure $2 \mathrm{~g}$ and $\mathrm{h}$ ). Next, to determine the functionality of SSG3 cells, we quantified the ratio of $\Delta 6 / \Delta 9$ desaturase that is an index of sebocyte maturation and associated metabolic process [35]. We found that this ratio in SSG3 cells is largely superior to the NIKS (178.868 and 11.424 respectively) reflecting the functionality of the scalp-derived sebocytes (Figure $2 \mathrm{~g}$ ). The lipid analysis also revealed that only fatty acids with evennumbered carbon chains, a characteristic of in vivo sebum, are present in SSG3 (Figure 2h). We conclude 

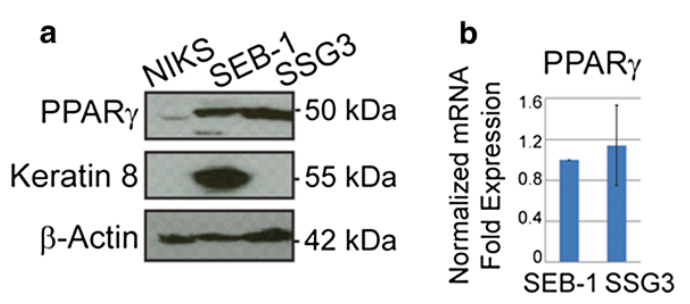

d
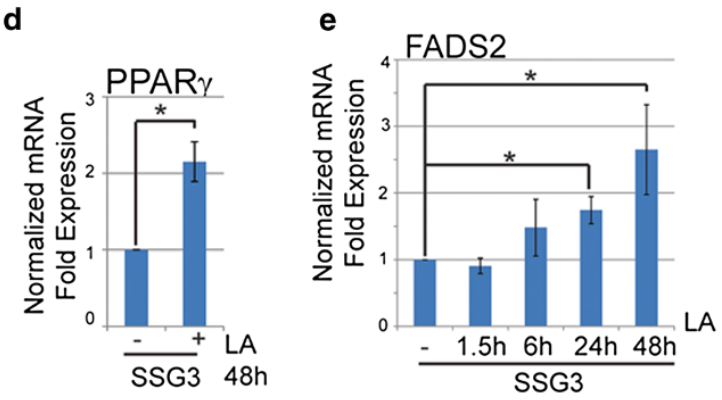

g

\begin{tabular}{|c|c|c|}
\cline { 2 - 3 } \multicolumn{1}{c|}{} & \multicolumn{1}{c|}{ NIKS } & \multicolumn{1}{c|}{ SSG3 } \\
\hline Sapienic acid* & $0.795 \%$ & $2.150 \%$ \\
\hline Palmitoleic acid** & $6.959 \%$ & $1.202 \%$ \\
\hline$\Delta 6 / \Delta \mathbf{9}$ & 11.424 & 178.868 \\
\hline
\end{tabular}

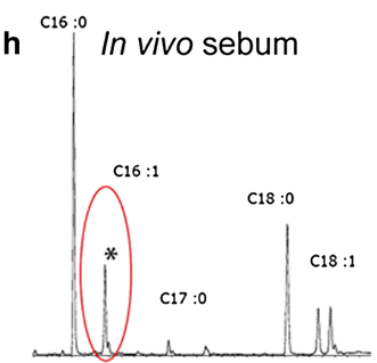

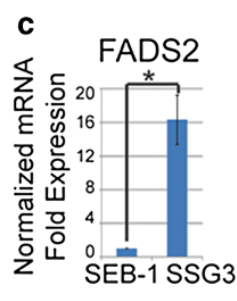

$f$

Figure 2 Primary sebocytes isolated from scalp sebaceous glands can differentiate in vitro and produce sebum-characteristic lipids. (a) SSG3 expresses PPARy but not Keratin 8 in contrast to SEB-1. (b-c) Real-time PCR shows that PPARY is equally expressed in SEB-1 and SSG3 whereas FADS2 is more highly expressed in SSG3 cells than SEB-1. RNA from SEB-1 and SSG3 derived from the scalp explant at passage 3 were normalized to GAPDH expression. Data shown represent three independent experiments each performed in triplicate (mean $+/-S D$, $n=3$ ).

${ }^{*}$ p-value $<0.05$ (unpaired two-tailed student's $t$ test). (d) Cells were cultivated for $48 \mathrm{~h}$ with or without $0.1 \mathrm{mM}$ of linoleic acid (LA). Differentiation through LA activation is followed by an increase in PPARY expression in SSG3 cells. * p-value <0.05 paired two-tailed student's $t$ test). (e) $24 \mathrm{~h}$ and $48 \mathrm{~h}$ of LA treatment induce a significant increase of FADS2 expression in SSG3 cells. ${ }^{*}$ p-value $<0.05$ (paired two-tailed student's $t$ test). (f) The $\Delta 6$ desaturase/FADS2 catalyzes the transformation of palmitic acid into sapienic acid. (g) Lipid analysis showing the percentage of $\Delta 9$ and $\Delta 6$ in the pellet of NIKS and SSG3 and the ratio $\Delta 6 / \Delta 9$. (h) The sapienic acid $\left(^{*}\right.$ ) can be detected in SSG3 as in vivo sebum, whereas in NIKS, the palmitoleic acid $\left(^{* *}\right)$ is the abundant lipid detected.

that the primary human sebocyte cultures we have established not only express genes involved in sebum production and lipid synthesis but can also produce sebum-specific lipids. We next investigated the mechanism by which cellular differentiation and lipid production are regulated in primary human sebocytes.

\section{TGF $\beta$ signaling is active in sebaceous gland in vivo and in vitro}

A previous study using whole sebaceous gland explants treated with various cytokines, suggested TGF $\beta$ as a potential candidate for human sebocyte regulation [19]. TGF $\beta$ ligands bind to a bidimeric receptor complex composed of TGF $\beta$ RI and TGF $\beta$ RII to phosphorylate and activate receptor-bound Smad (Smad2/3) transcription factors enabling them to translocate into the nucleus and regulate TGF $\beta$-responsive genes [36]. TGF $\beta$ RII is essential for the activation of the Smad2 pathway [23,37]. Therefore we analyzed the presence of TGF $\beta$ RII and the functionality of the pathway in vivo and in vitro by the presence of phosphorylated Smad2/3 as readout for TGF $\beta$ activation. Using immunofluorescence, we first verified that TGF $\beta$ RII is expressed throughout the sebaceous gland with the exception of the differentiated, lipid filled sebocytes present in the center of the gland (Figure 3a and 3a'). Further, we determined that the TGF $\beta$ pathway is active in the gland in vivo by detecting the expression of nuclear phosphorylated Smad2 in the undifferentiated and maturing sebocytes but not in terminally differentiated sebocytes present in the center of the gland (Figure $3 \mathrm{~b}$ and $3 \mathrm{~b}^{\prime}$ ). In vitro, Smad2 is phosphorylated in response to exogenously added recombinant TGF $\beta 1$ in SSG3 sebocytes, indicating the TGF $\beta$ pathway is intact and active in our in vitro system (Figure 3c). 


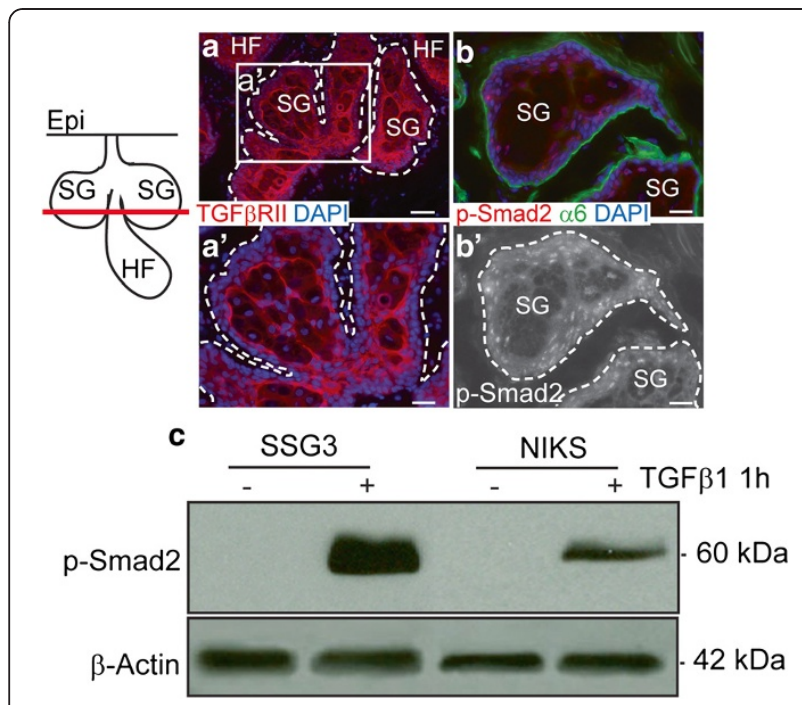

Figure 3 TGF $\beta$ signaling is active in sebaceous gland in vivo and in vitro. Sebaceous glands were sectioned in horizontal plane (red line in the diagram). (a) OCT sections of human scalp tissue stained with TGF $\beta$ RII (red) show expression of the receptor throughout the sebaceous gland with the exception of the differentiated cells in the center. Boxed area is magnified and shown to $\left(\mathbf{a}^{\prime}\right)$. (b) TGF $\beta$ pathway is active in vivo as denoted by the expression of nuclear phosphorylated Smad2 (red). a6: a6-integrin stains in green the basal layer of the sebaceous gland. Scale bars, 50 $\mu \mathrm{m}(\mathbf{a}), 20 \mu \mathrm{m}\left(\mathbf{a}^{\prime}, \mathbf{b}, \mathbf{b}^{\prime}\right)$. Abbreviations: Epi, Epidermis; HF, Hair Follicle; SG, Sebaceous Gland. (c) The indicated sebocyte cultures were treated with $5 \mathrm{ng} / \mathrm{ml}$ of TGF $\beta 1$ ligand for one hour and whole cell extracts were examined by immunoblot to determine the activation of the TGF $\beta$ pathway.

Effect of TGF $\beta$ signaling on sebocyte differentiation genes We next probed the effect of TGF $\beta$ signaling on their differentiation, by examining the expression of genes involved in lipogenesis upon treatment with TGF $\beta 1$. As shown in Figure $4 \mathrm{a}$ and b, when cells are stimulated with TGF 31 for $24 \mathrm{~h}$, the mRNA expression of FADS2 and $P P A R \gamma$ are significantly decreased in SSG3 cells suggesting that TGF $\beta 1$ may prevent cell differentiation. Similar results were obtained in primary sebocytes derived from breast and face (Additional file 3: Figure S3), suggesting that the response to TGF $\beta$ is indicative of sebocytes in general and not due to the skin tissue type. To test if these effects are dependent on the canonical TGF $\beta$ pathway, we used shRNA to knockdown TGF $\beta$ receptor II, thus effectively inhibiting Smad2 phosphorylation [23]. TGF $\beta$ RII expression was similarly reduced in SSG3 cells using two independent TGF $\beta$ RII shRNA (Figure 4c). Phosphorylated-Smad2 was decreased in shRNA expressing cells compared to controls after TGF $\beta$ activation (Figure $4 \mathrm{~d}$ ), as expected. We also detected a decrease of TGF $\beta$ RII in control cells treated with TGF $\beta 1$ for $24 \mathrm{~h}$ (Figure $4 \mathrm{c}$ ) reflecting the possible degradation of the receptor [38]. Moreover, the reduced TGF $\beta$ RII expression inhibited the ability of SSG3 cells to significantly decrease FADS2 and PPARy gene expression when cells are treated with TGF $\beta 1$ (Figure 4e and $f$ ). Our results indicate that the TGF $\beta$ pathway can directly control the expression of genes required for the differentiation of sebocytes.

Next we have determined how the inhibition of TGF $\beta$ signaling affects the functionality of SSG3 cells at a cellular level by analyzing the presence of cytoplasmic lipids in SSG3 shRNA expressing cells with reduced TGF $\beta$ RII. TGF $\beta$ RII depletion is associated with the increase of lipid inclusions positively stained with Nile red, Oil red $\mathrm{O}$, and identified by electron microscopy compared to SSG3 cells expressing a shRNA control (Figure 5b and c and Additional file 4: Figure S4). The lipid droplets labeled with Nile red were analyzed by flow cytometry (Figure 5d). Similar to cells treated with linoleic acid, an increase in fluorescence and granularity (representing the lipid droplets) of the cells was detected in SSG3 TGF $\beta$ RII shRNA expressing cells compared to the shRNA control.

Additionally, we found that whereas TGF $\beta 1$ treatment has no effect on the lipid production in the shRNA cells (Figure 5b), it induces a decrease in lipid inclusion in SSG3 infected with a non-targeting shRNA control (Figure 5a). These results suggest that inhibition of FADS2 and PPARY at the transcriptional level is mediated via canonical Smad signal transduction. Together, our findings show that activation of the TGF $\beta$ signaling pathway down-regulates the expression of genes involved in the production of characteristic sebaceous lipids. We found that TGF $\beta$ RII gene, which is essential for the activation of the Smad2 pathway, limits lipid production in primary human sebocytes. These findings illustrate the role of TGF $\beta$ in maintaining human sebocytes in an undifferentiated state by inhibiting their differentiation and highlight the relevance of this pathway in human sebaceous gland biology.

\section{Discussion}

Here we have developed a novel method of culturing human sebocytes without transformation and using a feeder layer-free culture system to examine the role of the TGF $\beta$ pathway in the control of differentiation. Primary sebaceous gland cells do not express Keratin 8 in contrast to previously immortalized sebocytes. Keratin 8 is not normally expressed in normal sebaceous gland in vivo [29] and our results indicate that the transformation process in the immortalized line has likely altered the expression of several fundamental cell markers. Moreover, we showed different responsiveness to linoleic acid and TGF $\beta 1$ treatment between the primary sebocytes and the immortalized cells (data not shown) suggesting that the cellular properties of those cells substantially differ.

Through our analysis, we have identified that certain markers of sebocytes are differentially expressed depending 

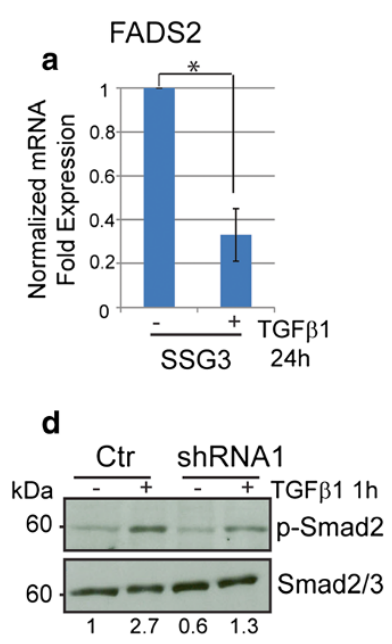
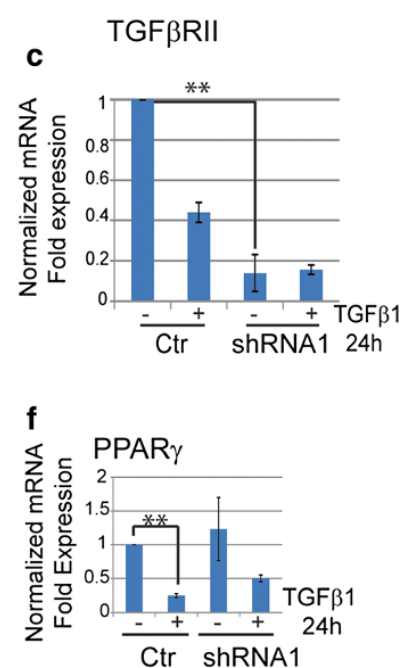

Figure 4 TGF $\beta$ signaling triggered decreased expression of lipogenic genes through the TGF $\beta$ RII-Smad2 dependent pathway.

(a, b) SSG3 cells were treated with $5 \mathrm{ng} / \mathrm{ml}$ of TGF 1 for 24 hours and used for qPCR. Data were normalized to GAPDH expression and relative expression determined using untreated cells as a reference. FADS2 and PPARY expression were found to be significantly downregulated in response to TGF $\beta 1$ treatment in SSG3 cells. (c) TGF $\beta$ R/l expression in SSG3 cells expressing TGF $\beta$ RI/ shRNA1 and a control shRNA (Ctr) shows the efficiency of the knockdown. (d). Immunoblot confirms the decrease of p-Smad2 activity in shRNA expressing cells stimulated with TGF $\beta 15$ ng/ml for $1 \mathrm{~h}$. Values, noted below the immunoblot, represent the relative density quantified with ImageJ using the ratio p-Smad2/Smad2/3 from each condition. (e, f). Decrease of FADS2 and PPARY at the transcriptional level is mediated via canonical Smad signal transduction. The expression was normalized to control (Ctr) untreated. The significant decrease in PPARY and FADS2 genes in control SSG3 cells after treatment with TGF 31 , is not detected in TGF $\beta$ RII-deficient SSG3 cells. ${ }^{*} \mathrm{p}$-value $<0.05,{ }^{* *} \mathrm{p}$-value $<0.001$ (paired two-tailed Student's t test).

upon the location on the body (scalp, chest, face), and localization within the sebaceous gland. These results highlight the need for studies covering a range of patient ages to fully comprehend the regulation of the sebaceous glands. However, our work shows that the effect of TGF $\beta 1$ activation on sebocyte differentiation is similar in sebocytes derived from three areas (scalp, breast and face) suggesting the specificity of that effect is independent of location. Previous reports have largely focused on cells and glands derived from older adults and post-menopausal women [14-16]. While we have not identified differences in sex, the age of the individual from which the sebaceous gland is derived seems to be of significance. It is known that the sebaceous glands undergo dramatic changes over the course of one's lifespan, with high sebum production occurring in infancy, a reduction during early childhood, followed by a steady increase through puberty into early adulthood. Using pediatric donors we ensured that the skin is not exposed to the hormonal changes that adult or old donor skin goes through. In the future it may be interesting to use our novel method to isolate sebocytes from old donors to examine the effect of age on TGF $\beta$ responsiveness in sebocytes.

We have begun to unravel one mechanism of differentiation of human sebaceous glands that culminates in sebum production. Our data suggest that TGF $\beta$ signaling maintains sebocytes in an undifferentiated state by decreasing the expression of FADS2 and PPARY thereby decreasing lipid accumulation through the TGF $\beta$ RIISmad2 dependent pathway. The successful growth of these primary human sebocytes has important clinical application such as the possibility of designing new strategies of culturing engineered skin to enable and maintain the presence of sebaceous glands in skin grafts for burn victims [10,39]. In addition to cell autonomous regulators and signals inducing proliferation and maturation among sebaceous cells, the complex microenvironment surrounding the sebaceous gland might have a profound effect on homeostasis of the tissue. Molecular crosstalk between the dermis and the epithelial cells is crucial for the initiation and maintenance of the hair follicles [40]. It seems most likely that similar mechanisms of communication between sebocytes and the surrounding dermal tissue exist. For instance, in the mouse, TGF $\beta 1$ is known to be released by the inner root sheath of the hair follicle, thereby providing a means for a bidirectional interaction between the sebaceous gland and the hair follicle epithelium [20]. Similarly, in the dermis, human fibroblasts secrete TGF $\beta[41,42]$ which may then act on keratinocytes and sebocytes. Another component in the microenvironment that could also be part of this crosstalk are the arrector pili muscle cells recently shown to be controlled by bulge stem cells in mouse [43]. Being located in close proximity to the sebaceous gland, arrector pili muscles could help release sebum onto the skin surface [44]. 

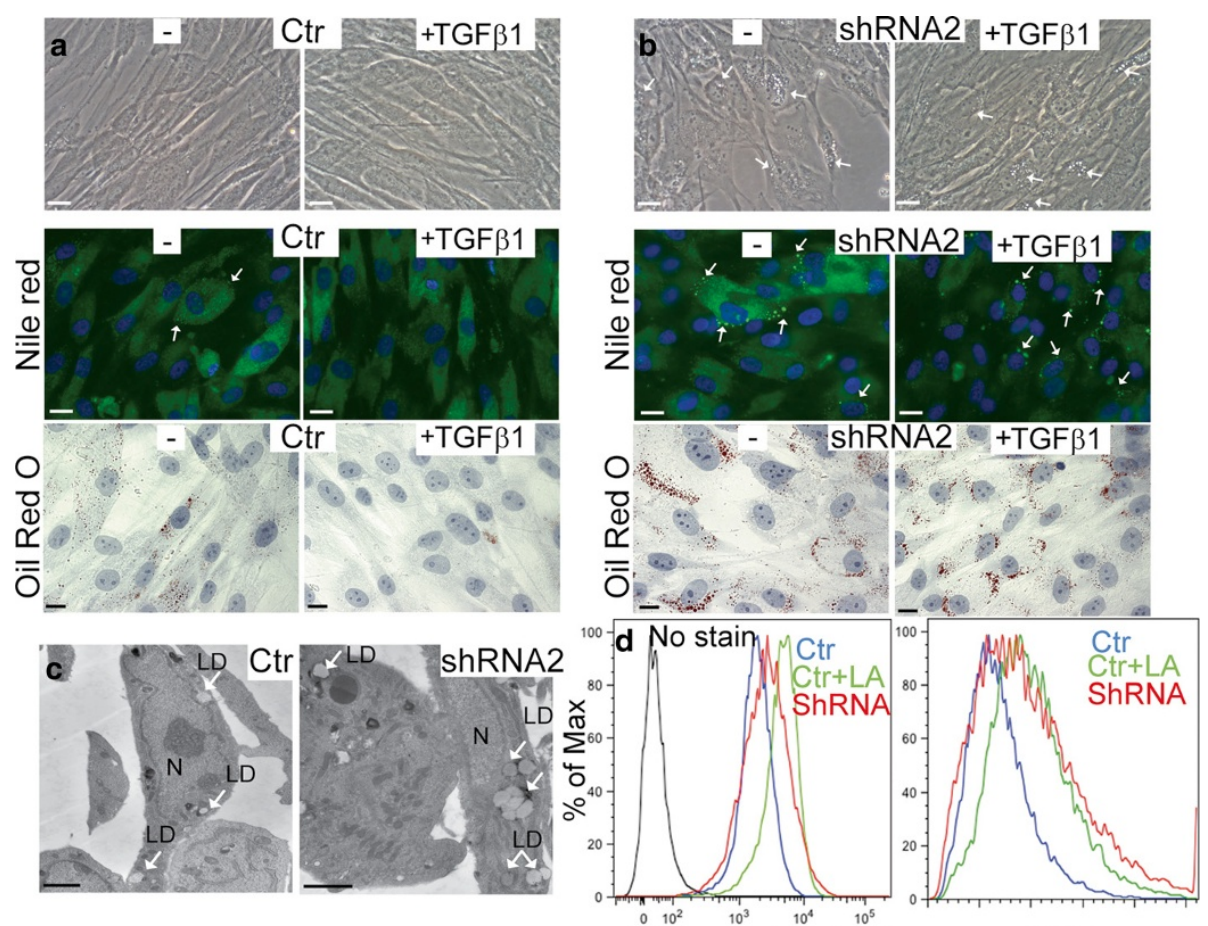

FL1- neutral lipids

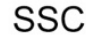

Figure $\mathbf{5}$ Inhibition of TGF $\beta$ signaling induces lipogenesis in primary SSG3 cells. (a, b) SSG3 cells stably expressing a shRNA against TGF $\beta$ RIl, show accumulation of lipid droplets on brightfield images (scale bars, $20 \mu \mathrm{m}$ ), by Nile red (scale bars, $20 \mu \mathrm{m}$ ) and Oil red O stainings (scale bars, $10 \mu \mathrm{m}$ ). White arrows show the presence of multiple lipid droplets in the shRNA expressing cells compared to the control (Ctr). $24 \mathrm{~h}$ of TGF $\beta 1(5 \mathrm{ng} / \mathrm{ml})$ treatment decreases the basal level of lipid production in control cells but does not affect cells expressing the TGF $\beta$ R/l shRNA, mainly seen by Oil red O. (c) Electron microscopy showing the increase of lipid droplets in SSG3 cells (denoted by white arrows) expressing the shRNA compared to the control. Scale bars, 2 m. LD, Lipid Droplets. N, Nucleus. (d) Flow cytometry of SSG3 cells expressing the shRNA labeled with Nile red. FL-1 measures the neutral lipids and SSC reflects the granularity of the cells. 10,000 cells have been acquired for each condition. As a positive control, SSG3 treated by $0.1 \mathrm{mM}$ linoleic acid (LA) for $24 \mathrm{~h}$ show increase of fluorescence and granularity representing the lipid droplets. Note the increase of fluorescence and the increase of granularity in shRNA expressing TGF $\beta$ RIl compared to the cells expressing a shRNA control. We obtained similar result with two different shRNA expressing TGF $\beta$ RIl (Additional file 4: Figure S4).

Impairment of the skin barrier due to the deregulation of sebum production when associated with bacteria colonization and inflammation, can be the cause of serious skin conditions in people. For instance, hyperseborrhea combined with the presence of Propionibacterium acnes and inflammation can lead to acne vulgaris [4] and Staphylococcus aureus can aggravate atopic dermatitis [6]. Sebocytes can produce antimicrobial peptides such as defensin-1 and -2 upon exposure to Propionibacterium acnes or lipopolysaccharides $[45,46]$ to prevent from bacteria colonization [47] and from an upregulation of sebum production [48]. Studies have revealed that TGF $\beta$ induces the expression of human $\beta$-defensin- 2 in endothelial cells [49] and influences inflammatory response [50]. Therefore it will be interesting to further investigate the impact of TGF $\beta$ on immune responses in sebaceous gland and its implication in antimicrobial peptides secretion by sebocytes. With the novel isolation strategy we described here, different interactions with the microenvironment can now be investigated.

\section{Conclusions}

By describing an innovative way to grow and successfully passage human primary sebocytes, we have overcome a major hurdle in the field of epithelial cell culture. We characterized the role of TGF $\beta$ signaling pathway in the inhibition of lipogenesis in these cells by showing that reduced expression of TGF $\beta$ RII increases lipid production. Our work, can not only improve our understanding of the physiology of the sebaceous gland in normal and pathological conditions [45] but also potentially expand this knowledge to other glands like eccrine and apocrine glands and use these cells to improve the quality of the skin grafts.

\section{Methods}

\section{Cell Culture}

The sebaceous gland populations were generated from human scalp (SSG3), face, chest and breast from both male and female donors. The skin samples were collected as a surgical waste with information provided 
regarding the age and sex of the donors with Institutional Review Board (IRB) approval at Cincinnati Children's Hospital Medical Center. Cincinnati Children's Hospital is a Pediatric Hospital that allowed us to collect samples from donors ranging 9 months old to 12 years old. The IRB determined that the research does not meet the regulatory criteria for research involving human subjects as there were no interaction with the donors and no identifiable private information. After treating the skin with dispase overnight at $4{ }^{\circ} \mathrm{C}$, intact sebaceous glands were isolated with microsurgical instruments under a dissecting microscope (Figure 1b). To mimic the microenvironment of the sebaceous gland, the explants were sandwiched between glass coverslips coated with human fibronectin (10 $\mu \mathrm{g} / \mathrm{ml}$, Millipore, Billerica, MA) (Figure 1d). The explants were cultivated in sebocyte medium as described [15] (DMEM/Ham's F-12 (3:1), Epidermal Growth Factor (EGF $3 \mathrm{ng} / \mathrm{ml}$, Austral Biologicals, San Ramon, CA), cholera toxin (1.2×10-10M, Sigma, St. Louis, MO), adenine $(24 \mu \mathrm{g} / \mathrm{ml}$, Sigma, St. Louis, MO), insulin (10 ng/ $\mathrm{ml}$ Sigma), hydrocortisone (45.2 ng/ml, Sigma), FBS (2.5\% Hyclone, San Jose, CA), antibiotic/antimycotic (100x, Invitrogen, Grand Island, NY). After 1-2 weeks of growth in culture, cellular outgrowth became apparent from the periphery of the gland lobules. The explants were removed and the isolated cells cultured on the fibronectin-coated coverslips.

\section{Western blotting}

Proteins were separated by electrophoresis on $8-10 \%$ acrylamide gels, transferred to nitrocellulose membranes and subjected to immunoblotting. Membranes were blocked for one hour with $5 \%$ non-fat milk or $5 \%$ BSA in PBS containing $0.1 \%$ Tween-20. Primary antibodies were used at concentrations described below and HRPcoupled secondary antibodies were used at $1 / 2,000$ in $5 \%$ non-fat milk. Immunoblots were developed using standard ECL (Amersham, Pittsburg, PA) and Luminata TM crescendo and classico (Millipore, Billerica, MA). Two-color immunoblot detection was performed using LI-COR Odyssey CL× (Biosciences, Lincoln, NE). Membranes were blocked in Odyssey blocking buffer (Li-Cor) and secondary antibodies conjugated to IRDye 680LT and $800 \mathrm{CW}$ were used (1/10,000; Li-Cor). Protein levels were quantified using the Odyssey Infrared Imaging System (Li-Cor).

\section{Retroviral Infection}

To ablate TGF $\beta$ RII in SSG3 cells, we used shRNA vectors from the CCHMC Heart Institute lenti-shRNA library core (shRNA TGF $\beta$ RII \#197031 and 194992 and a shRNA control). The human library was purchased from Sigma-Aldrich (MISSION shRNA). Lentivirus was produced by the Viral Vector Core at the Translational Core
Laboratories, Cincinnati Children's Hospital Research Foundation. Cells were grown to $80 \%$ confluency in 6well plates before being infected with the lentivirus for $48 \mathrm{~h}$. Infected cells were selected with $1 \mu \mathrm{g} / \mathrm{ml}$ puromycin (Sigma, St. Louis, MO) for 48 h. Following selection, TGF $\beta$ RII knock down cells were grown in regular media for $48 \mathrm{~h}$ before being activated with $5 \mathrm{ng} / \mathrm{ml}$ TGF $\beta 1$ for $24 \mathrm{~h}$.

\section{Histology and Immunofluorescence}

Human tissues were frozen unfixed in OCT compound (Tissue-Tek, Sakura, Torrance, CA) for cryosectioning. Immunostainings were performed as previously described [51].

\section{Antibodies}

Primary antibodies against the following proteins were used at the dilution indicated: PPARY (Santa-Cruz Biotechnology Inc., Santa Cruz, CA, H-100 1/250 for immunofluorescence, 1/500 for immunoblot), Blimp1 (Cell Signaling Technology, Beverly, MA, 1/500 for immunofluorescence, $1 / 1,000$ for immunoblot), Fibronectin (Santa-Cruz Biotechnology Inc., Santa Cruz, CA, EP5 1/ 150), Muc1 (Millipore, Billerica, MA 1/500), cMyc (Cell Signaling Technology, Beverly, MA, 1/800 for immunofluorescence, 1/1,000 for immunoblot), TGF $\beta$ RII (SantaCruz Biotechnology Inc., Santa Cruz, CA, sc-220 1/ 1,000), p-Smad2 (Cell Signaling Technology, Beverly, MA, 1/100 for immunofluorescence, 1/1,000 for immunoblot), Smad2/3 (BD Biosciences, San Jose, CA, 1/500), $\alpha 6$ integrin (CD49f, BD Biosciences, San Jose, CA, 1/ 100), Keratin 8 (this antibody, developed by Dr. Brulet and Dr. Kemler, was obtained from the NICHD Developmental Studies Hybridoma Bank maintained by the University of Iowa, 1/1,000), $\beta$-actin (Sigma, St. Louis, MO, 1/2,000), Keratin 7 (Cell Signaling Technology, Beverly, MA, 1/1,000), 4',6-diamidino-2-phenylindole (DAPI) was utilized as a marker of cell nuclei (Sigma Chemical Co., St. Louis, MO, 1/5,000). Secondary antibodies Alexa Fluor 488 or 555 (Molecular Probes, Grand Island, NY) were used at a dilution of $1 / 1,000$. Fluorescence images were acquired with a fluorescent microscope AxioImager M1 (Zeiss, Oberkochen, Germany) and pictures were taken with an axioCam MRm camera (Zeiss, Oberkochen, Germany).

\section{Real-time PCR}

Total RNA was isolated using a Qiagen Rneasy Mini Kit and used to produce cDNA (Maxima first strand cDNA synthesis kit, Fermentas, San Jose, CA). Reverse transcription (RT) reactions were diluted to $10 \mathrm{ng} / \mu \mathrm{l}$ and $1 \mu \mathrm{l}$ of each RT was used for real-time PCR. Real-time PCR was performed using the CFX96 real-time PCR System, CFX Manager Software and the SsoFast EvaGreen 
Supermix reagents (Biorad, Hercules, CA). All reactions were run in triplicate and analyzed using the $\triangle \Delta C T$ method with relative expression normalized to GAPDH. Primers used:

GAPDH-F: ACATCGCTCAGACACCATG, GAPDHR: TGTAGTTGAGGTCAATGAAGGG

PPAR $\gamma$-F: GAGCCCAAGTTTGAGTTTGC, PPAR $\gamma$-R: GCAGGTTGTCTTGAATGTCTTC,

FADS2-F: TGTCTACAGAAAACCCAAGTGG, FADS

2-R: TGTGGAAGATGTTAGGCTTGG,

TGF $\beta$ RII-F: CTGTGGATGACCTGGCTAAC, TGF $\beta$ RII-R: CATTTCCCAGAGCACCAGAG

\section{Lipogenesis assays}

For Nile red staining, cells or OCT sections were fixed 10 minutes at room temperature in $4 \%$ formaldehyde. After 3 washes in 1XPBS, Nile red staining was performed with $0.1 \mu \mathrm{g} / \mathrm{ml}$ of Nile red (Sigma, St. Louis, MO) in 0.15 $\mathrm{M} \mathrm{NaCl}$ for 15 minutes at room temperature. For Oil red O staining, cells were fixed 15 minutes in $10 \%$ formalin, wash with water for 10 minutes and 60\% isopropanol before being stained with Oil red O $(0.7 \%$ in $60 \%$ isopropanol) for 45 minutes. Cells were rinsed with $60 \%$ isopropanol and the nuclei stained with haematoxylin. To trigger differentiation of sebocytes in vitro, $0.1 \mathrm{mM}$ linoleic acid (Sigma, St Louis, MO) was added directly to sebocyte media. To prepare cells for extraction of lipids, $2-3 \times 10^{7}$ of cells were pelleted, washed with 1 XPBS and lipids were preserved in the dark at $-80^{\circ} \mathrm{C}$ under argon until analysis. The qualitative and quantitative composition of lipids in scalp-derived human sebocytes was determined using an Agilent 5973N Gas chromatograph/Mass spectrometer with a SPE cartridge (solid phase extraction) and was performed by Synelvia S.A.S (Labege, France).

\section{Nile Red analysis by FACS}

Cells were cultured in 6-well plates at $80 \%$ confluence and infected with the lentivirus expressing the shRNAs as previously described. After puromycin selection for $48 \mathrm{~h}$, cells were washed in $1 \mathrm{X}$ PBS and treated with working medium with or without Linoleic acid $(0.1 \mathrm{mM})$ for $24 \mathrm{~h}$. The cells were trypsinized, washed once with 1X PBS and neutral lipids were labeled with the fluorescent dye Nile red ( $1 \mu \mathrm{g} / \mathrm{ml}$ in PBS). 10,000 cells per sample were analyzed using a FACS Canto I equipped with a blue laser (488 $\mathrm{nm}$ excitation).

\section{Electron microscopy}

Cells were grown at $80 \%$ confluency in sebocyte media and rinsed once with $0.175 \mathrm{M}$ sodium cacodylate buffer. Cells were fixed in $3 \%$ glutaraldehyde/0.175 M cacodylate buffer (Electron Microscopy Sciences, Hatfield, PA) for 1 hour at $4^{\circ} \mathrm{C}$. Dishes were washed twice with 0.175 $\mathrm{M}$ sodium cacodylate buffer. Cells were post fixed in 1\% osmium tetroxide/cacodylate buffer for 1 hour at $4^{\circ} \mathrm{C}$ before being washed three times with $0.175 \mathrm{M}$ sodium cacodylate buffer. After the final wash with $1.5 \mathrm{ml}$, cells were scraped and centrifuged for 5 min at 10,000 RPM. The cell pellet was then resuspended in $1 \mathrm{ml} 1 \%$ agarose (Type IX ultra-low gelling tempt, Sigma) overnight at $4^{\circ} \mathrm{C}$. The samples were then processed through a graded series of alcohols, infiltrated and embedded in LX-112 resin. After polymerization at $60^{\circ} \mathrm{C}$ for three days, ultrathin sections $(100 \mathrm{~nm})$ were cut using a ReichertJung Ultracut E microtome and counterstained in $2 \%$ aqueous uranyl acetate and Reynolds lead citrate. Images were taken with a transmission electron microscope (Hitachi H-6750) equipped with a digital camera (AMT $2 \mathrm{k} \times 2 \mathrm{~K}$ tem $\mathrm{CCD})$.

\section{Statistics}

Data are expressed as means +/- SD. Comparison between two cell types was performed using unpaired twotailed student's $t$ test. Paired two-tailed student's $t$ test was used when we compared the effect of a treatment on the same cell type. $\mathrm{p}<0.05$ was considered significant.

\section{Additional files}

\begin{abstract}
Additional file 1: Figure S1. Primary human sebocytes derived from scalp, breast, chest and face tissues express typical sebocyte markers. (a) Hematoxylin and Eosin staining of the scalp sample. Scale bar, $50 \mu \mathrm{m}$. (b) Immunofluorescence staining showed that PPARY (red) is expressed in human sebaceous glands from the scalp explant at the periphery stained with a6-integrin (green) and at the center of the gland. Scale bar, $50 \mu \mathrm{m}$. Boxed area is magnified and shown to ( $\left.b^{\prime}\right)$. (c) Blimp1 (red) expression is mostly found in the differentiated cells of the sebaceous gland and in the inner root sheath of the hair follicle. a6-integrin (green) marked the basal layer of the gland. (d) Keratin 7 (red) expression varies depending on the location of the gland (scalp, breast and chest) as shown by immunofluorescence. (e-g) Sebocytes derived from the scalp, breast, chest and face explants expressed sebocytes markers by two-color immunoblot (Blimp1, c-Myc, Muc1, PPARY and K7). SSG4 represents primary sebocytes derived from a four year old-scalp sample. Scale bars, $50 \mu \mathrm{m}$ (b), $50 \mu \mathrm{m}$ (c and d). Abbreviations: SG, Sebaceous Gland; HF, Hair Follicle; a6, a6-integrin; K7, Keratin 7.
\end{abstract}

Additional file 2: Figure S2. Primary sebocytes can differentiate in vitro. (a) Human scalp sections showing evidence of lipid accumulation (Nile red stain). Scale bar, $50 \mu \mathrm{m}$ (b) SSG3 cells derived from the scalp explants were treated with $0.1 \mathrm{mM}$ linoleic acid (LA) for $48 \mathrm{~h}$ to differentiate the cells and stained with Nile red to detect lipids. Images were taken with the same exposure time in untreated and linoleic acidtreated conditions. Brightfield pictures showed accumulation of cytoplasmic lipid droplets after linoleic acid treatment as denoted by the black arrows. Scale bars, $50 \mu \mathrm{m}$ (c) Electron microscopy showing cytoplasmic lipid droplets in untreated sebocytes SSG3 derived from the scalp explants. Scale bar, $20 \mu \mathrm{m}$. Boxed area is magnified and shown to (c') scale bar, $500 \mathrm{~nm}$. (c') After linoleic acid treatment increased highelectron density lipid droplets are detected in SSG3 cells and magnified in $C^{\prime \prime \prime}$. Scale bars for $C^{\prime \prime}$ and $C^{\prime \prime \prime}$ are $2 \mu \mathrm{m}$. Abbreviations: HF, Hair Follicle. SG, Sebaceous Gland. LD, Lipid Droplets. N, Nucleus. Mi, Mitochondria. RER, Rough Endoplasmic Reticulum . SER, Smooth Endoplasmic Reticulum.

Additional file 3: Figure S3. TGF $\beta$ signaling triggered decreased expression of lipogenic genes in breast and face-derived sebocytes. RNA was isolated from sebocytes-derived from breast and face untreated or 
treated with $5 \mathrm{ng} / \mathrm{ml}$ of TGF $\beta 1$ for $24 \mathrm{~h}$ and used for real-time PCR. Two experiments were performed and all GPCR reactions were performed in triplicate. Data were normalized to GAPDH expression for each cell population and changes in relative expression were determined using untreated cells as a reference point. (a) FADS2 and (b) PPARY expression was found to be decreased significantly in response to TGF $\beta 1$ treatment as shown in scalp-derived sebocytes (Figure 4a-b) suggesting that the inhibitory effect of TGF $\beta$ is not due to the skin tissue type. ${ }^{*} p$-value $<0.05$ (paired two-tailed Student's $t$ test).

Additional file 4: Figure S4. Inhibition of TGF $\beta$ signaling induces lipogenesis in primary SSG3 cells. (a) SSG3 cells, stably expressing a shRNA against TGF $\beta$ RII (shRNA1), show accumulation of lipid droplets on brightfield image (white arrows) and by Nile red staining (shown in green) compared to cells infected with shRNA control. Scale bars, $20 \mu \mathrm{m}$. (b-c), Electron microscopy showing the increase of lipid droplets in SSG3 cells (denoted by white arrows) expressing the shRNA against TGF $\beta$ RII (shRNA2) compared to the control. Myelin figures, which indicate lipids synthesis, are detected in SSG3 cells expressing the shRNA. Abbreviations: $\mathrm{N}$, nucleus. LD, Lipid Droplets. Scale bars for $\mathrm{b}$ and $\mathrm{c}$ are $2 \mu \mathrm{m}$ and 500 $\mathrm{nm}$ for $\mathrm{c}^{\prime}$.

\section{Competing interests}

GG has no conflict of interest. JD, CB and LB are employed by L'Oreal and have conflict of interests.

\section{Authors' contributions}

$A J M, Y D, J D, M B, C B, L B$ and $G G$ contributed to experimental design. AJM, $Y D$ and $M B$ performed the experiments. CBG and AUR provided the human samples and advised on tissues preparation. PFL provides the NIKS cells. $A J M, Y D, J D, M B, L B$ and $G G$ assisted in data analysis. GG wrote and prepared the manuscript with critical comments from all authors. GG and LB have contributed equally to supervise the project. All authors read and approved the final manuscript.

\section{Acknowledgements}

We would like to thank Dr. Diane Thiboutot (Penn State) for providing the SEB-1 cells. Laura Runck (Guasch Lab) for helping with the histology of the sections. Georgianne Ciraolo (CCHMC Pathology Department) for performing the preparation of the samples for the electron microscopy, Xiaoling Zhang and Monica Delay (CCHMC Flow Cytometry Core) for support with the analysis of the Nile Red, and the viral vector core (CCHMC) for producing the lentivirus. Drs. Christopher Wylie and Susanne Wells for critical reading of the manuscript. This work was supported by L'Oreal.

\section{Author details}

'Division of Developmental Biology, Cincinnati Children's Hospital Medical Center, 3333 Burnet Avenue, Cincinnati, OH 45229, USA. ${ }^{2}$ Current Address: Department of Biomedical Sciences, College of Veterinary Medicine, Cornell University, Ithaca, NY 14853, USA. ${ }^{3}$ Current Address: Department of Dermatology, Columbia University, College of Physicians and Surgeons, New York, NY 10032, USA. “'L'OREAL Research \& Innovation, 90 rue du General Roguet, 92583, CLICHY, FRANCE. ${ }^{5}$ Division of Plastic Surgery, Children's Hospital Medical Center, 3333 Burnet Avenue, Cincinnati, OH 45229, USA. ${ }^{6}$ University of Wisconsin School of Medicine and Public Health, Madison, WI, USA.

Received: 13 September 2012 Accepted: 18 January 2013

Published: 23 January 2013

\section{References}

1. Thody AJ, Shuster S: Control and function of sebaceous glands. Physiological reviews 1989, 69(2):383-416.

2. Hyman AB, Brownstein MH: Tyson's "glands." Ectopic sebaceous glands and papillomatosis penis. Archives of dermatology 1969, 99(1):31-36.

3. Smith KR, Thiboutot DM: Thematic review series: skin lipids. Sebaceous gland lipids: friend or foe? J Lipid Res 2008, 49(2):271-281.

4. Picardo M, Ottaviani M, Camera E, Mastrofrancesco A: Sebaceous gland lipids. Dermatoendocrinol 2009, 1(2):68-71

5. Pochi PE, Strauss JS, Downing DT: Age-related changes in sebaceous gland activity. J Invest Dermatol 1979, 73(1):108-111.
6. Huang JT, Abrams M, Tlougan B, Rademaker A, Paller AS: Treatment of Staphylococcus aureus colonization in atopic dermatitis decreases disease severity. Pediatrics 2009, 123(5):e808-e814.

7. Stenn KS: Insights from the asebia mouse: a molecular sebaceous gland defect leading to cicatricial alopecia. J Cutan Pathol 2001, 28(9):445-447.

8. Zheng Y, Eilertsen KJ, Ge L, Zhang L, Sundberg JP, Prouty SM, Stenn KS, Parimoo S: Scd1 is expressed in sebaceous glands and is disrupted in the asebia mouse. Nat Genet 1999, 23(3):268-270.

9. Shizuru JA, Negrin RS, Weissman IL: Hematopoietic stem and progenitor cells: clinical and preclinical regeneration of the hematolymphoid system. Annu Rev Med 2005, 56:509-538.

10. Ronfard V, Rives JM, Neveux Y, Carsin H, Barrandon Y: Long-term regeneration of human epidermis on third degree burns transplanted with autologous cultured epithelium grown on a fibrin matrix. Transplantation 2000, 70(11):1588-1598.

11. Barrandon $Y$, Green H: Three clonal types of keratinocyte with different capacities for multiplication. Proc Natl Acad Sci U S A 1987, 84(8):2302-2306.

12. Barrandon Y: Crossing boundaries: stem cells, holoclones, and the fundamentals of squamous epithelial renewal. Cornea 2007, 26(9 Suppl 1):S10-S12.

13. Abdel-Naser MB: Selective cultivation of normal human sebocytes in vitro; a simple modified technique for a better cell yield. Exp Dermatol 2004, 13(9):562-566.

14. Zouboulis CC, Seltmann H, Neitzel H, Orfanos CE: Establishment and characterization of an immortalized human sebaceous gland cell line (SZ95). J Invest Dermatol 1999, 113(6):1011-1020.

15. Thiboutot D, Jabara S, McAllister JM, Sivarajah A, Gilliland K, Cong Z, Clawson G: Human skin is a steroidogenic tissue: steroidogenic enzymes and cofactors are expressed in epidermis, normal sebocytes, and an immortalized sebocyte cell line (SEB-1). J Invest Dermatol 2003, 120(6):905-914.

16. Lo Celso C, Berta MA, Braun KM, Frye M, Lyle S, Zouboulis CC, Watt FM: Characterization of bipotential epidermal progenitors derived from human sebaceous gland: contrasting roles of c-Myc and beta-catenin. Stem Cells 2008, 26(5):1241-1252.

17. Xia L, Zouboulis CC, Ju Q: Culture of human sebocytes in vitro. Dermatoendocrinol 2009, 1(2):92-95.

18. Furue M, Kato M, Nakamura K, Nashiro K, Kikuchi K, Okochi H, Miyazono K, Tamaki K: Dysregulated expression of transforming growth factor beta and its type-I and type-II receptors in basal-cell carcinoma. Int I Cancer 1997, 71(4):505-509.

19. Downie MM, Sanders DA, Kealey T: Modelling the remission of individual acne lesions in vitro. Br J Dermatol 2002, 147(5):869-878.

20. Wollina $U$, Lange $D$, Funa $K$, Paus R: Expression of transforming growth factor beta isoforms and their receptors during hair growth phases in mice. Histol Histopathol 1996, 11(2):431-436.

21. Paus R, Foitzik $K$, Welker $P$, Bulfone-Paus $S$, Eichmuller $S$ : Transforming growth factor-beta receptor type I and type II expression during murine hair follicle development and cycling. J Invest Dermatol 1997, 109(4):518-526.

22. Pietenpol JA, Stein RW, Moran E, Yaciuk P, Schlegel R, Lyons RM, Pittelkow MR, Munger K, Howley PM, Moses HL: TGF-beta 1 inhibition of c-myc transcription and growth in keratinocytes is abrogated by viral transforming proteins with pRB binding domains. Cell 1990, 61(5):777-785

23. Guasch G, Schober M, Pasolli HA, Conn EB, Polak L, Fuchs E: Loss of TGFbeta signaling destabilizes homeostasis and promotes squamous cell carcinomas in stratified epithelia. Cancer Cell 2007, 12(4):313-327.

24. Arnold I: Watt FM: C-Myc activation in transgenic mouse epidermis results in mobilization of stem cells and differentiation of their progeny. Curr Biol 2001, 11(8):558-568.

25. Yang L, Wang L, Yang X: Disruption of Smad4 in mouse epidermis leads to depletion of follicle stem cells. Mol Biol Cell 2009, 20(3):882-890.

26. Rosenfield RL, Deplewski D, Kentsis A, Ciletti N: Mechanisms of androgen induction of sebocyte differentiation. Dermatology 1998, 196(1):43-46.

27. Allen-Hoffmann BL, Schlosser SJ, Ivarie CA, Sattler CA, Meisner LF, O'Conno $S L$ : Normal growth and differentiation in a spontaneously immortalized near-diploid human keratinocyte cell line, NIKS. J Invest Dermatol 2000, 114(3):444-455.

28. Tsubura A, Okada H, Sasaki M, Dairkee SH, Morii S: Immunohistochemical demonstration of keratins 8 and 14 in benign tumours of the skin appendage. Virchows Archiv A, Pathological anatomy and histopathology 1991, 418(6):503-507. 
29. Troyanovsky SM, Guelstein VI, Tchipysheva TA, Krutovskikh VA, Bannikov GA: Patterns of expression of keratin 17 in human epithelia: dependency on cell position. J Cell Sci 1989, 93(Pt 3):419-426.

30. Magnusdottir E, Kalachikov S, Mizukoshi K, Savitsky D, Ishida-Yamamoto A, Panteleyev AA, Calame K: Epidermal terminal differentiation depends on B lymphocyte-induced maturation protein-1. Proc Natl Acad Sci U S A 2007, 104(38):14988-14993.

31. Braun KM, Niemann C, Jensen UB, Sundberg JP, Silva-Vargas V, Watt FM: Manipulation of stem cell proliferation and lineage commitment: visualisation of label-retaining cells in wholemounts of mouse epidermis. Development 2003, 130(21):5241-5255.

32. Rosenfield RL, Kentsis A, Deplewski D, Ciletti N: Rat preputial sebocyte differentiation involves peroxisome proliferator-activated receptors. J Invest Dermatol 1999, 112(2):226-232.

33. Ge L, Gordon JS, Hsuan C, Stenn K, Prouty SM: Identification of the delta-6 desaturase of human sebaceous glands: expression and enzyme activity. J Invest Dermatol 2003, 120(5):707-714.

34. Pappas A, Anthonavage M, Gordon JS: Metabolic fate and selective utilization of major fatty acids in human sebaceous gland. J Invest Dermatol 2002, 118(1):164-171.

35. Ottaviani M, Camera E, Picardo M: Lipid mediators in acne. Mediators Inflamm 2010

36. Massague J, Gomis RR: The logic of TGFbeta signaling. FEBS Lett 2006 580(12):2811-2820

37. Wrana JL, Attisano L, Wieser R, Ventura F, Massague J: Mechanism of activation of the TGF-beta receptor. Nature 1994, 370(6488):341-347.

38. Mitchell H, Choudhury A, Pagano RE, Leof EB: Ligand-dependent and independent transforming growth factor-beta receptor recycling regulated by clathrin-mediated endocytosis and Rab11. Mol Biol Cell 2004, 15(9):4166-4178.

39. Eisinger M, Li WH, Rossetti DD, Anthonavage M, Seiberg M: Sebaceous gland regeneration in human skin xenografts. J Invest Dermatol 2010, 130(8):2131-2133

40. Rendl M, Lewis L, Fuchs E: Molecular dissection of mesenchymal-epithelial interactions in the hair follicle. PLOS Bio/ 2005, 3(11):e331.

41. Wong T, McGrath JA, Navsaria $H$ : The role of fibroblasts in tissue engineering and regeneration. Br J Dermatol 2007, 156(6):1149-1155.

42. Nolte SV, Xu W, Rennekampff HO, Rodemann HP: Diversity of fibroblasts-a review on implications for skin tissue engineering. Cells Tissues Organs 2008, 187(3):165-176.

43. Fujiwara H, Ferreira M, Donati G, Marciano DK, Linton JM, Sato Y, Hartner A, Sekiguchi K, Reichardt LF, Watt FM: The basement membrane of hair follicle stem cells is a muscle cell niche. Cell 2011, 144(4):577-589.

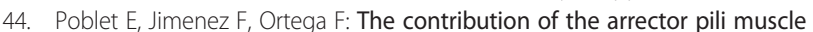
and sebaceous glands to the follicular unit structure. J Am Acad Dermatol 2004, 51(2):217-222.

45. Chronnell CM, Ghali LR, Ali RS, Quinn AG, Holland DB, Bull JJ, Cunliffe WJ, McKay IA, Philpott MP, Muller-Rover S: Human beta defensin-1 and -2 expression in human pilosebaceous units: upregulation in acne vulgaris lesions. J Invest Dermatol 2001, 117(5):1120-1125.

46. Nagy I, Pivarcsi A, Kis K, Koreck A, Bodai L, McDowell A, Seltmann H, Patrick $S$, Zouboulis CC, Kemeny L: Propionibacterium acnes and lipopolysaccharide induce the expression of antimicrobial peptides and proinflammatory cytokines/chemokines in human sebocytes. Microbes Infect 2006, 8(8):2195-2205.

47. Lee DY, Yamasaki K, Rudsil J, Zouboulis CC, Park GT, Yang JM, Gallo RL: Sebocytes express functional cathelicidin antimicrobial peptides and can act to kill propionibacterium acnes. J Invest Dermatol 2008, 128(7):1863-1866.

48. Zouboulis CC: Propionibacterium acnes and sebaceous lipogenesis: a love-hate relationship? J Invest Dermatol 2009, 129(9):2093-2096.

49. Kawsar HI, Ghosh SK, Hirsch SA, Koon HB, Weinberg A, Jin G: Expression of human beta-defensin-2 in intratumoral vascular endothelium and in endothelial cells induced by transforming growth factor beta. Peptides 2010, 31(2):195-201.
50. Ishinaga $H$, Jono $H$, Lim JH, Kweon SM, Xu H, Ha UH, Koga T, Yan C, Feng $\mathrm{XH}$, Chen LF, et al: TGF-beta induces p65 acetylation to enhance bacteriainduced NF-kappaB activation. EMBO J 2007, 26(4):1150-1162.

51. Runck LA, Kramer M, Ciraolo G, Lewis AG, Guasch G: Identification of epithelial label-retaining cells at the transition between the anal canal and the rectum in mice. Cell Cycle 2010, 9(15):3039-3045.

doi:10.1186/1471-5945-13-2

Cite this article as: McNairn et al:: TGF $\beta$ signaling regulates lipogenesis in human sebaceous glands cells. BMC Dermatology 2013 13:2.

\section{Submit your next manuscript to BioMed Central and take full advantage of:}

- Convenient online submission

- Thorough peer review

- No space constraints or color figure charges

- Immediate publication on acceptance

- Inclusion in PubMed, CAS, Scopus and Google Scholar

- Research which is freely available for redistribution

Submit your manuscript at www.biomedcentral.com/submit
C) BioMed Central 